\title{
Correction to: Geometric Continuum Mechanics
}

\author{
Reuven Segev and Marcelo Epstein
}

\section{Correction to:}

R. Segev, M. Epstein (eds.),

Geometric Continuum Mechanics, Advances in Mechanics and Mathematics 43, https://doi.org/10.1007/978-3-030-42683-5

This book has been inadvertently published with the volume number 42 which has now been modified as 43 . 